\title{
Modelling the variation in the behaviour of pre-fractured rocks subjected to hydraulic fracturing with permeability of the rock matrix using finite-discrete element method
}

\author{
Shahrzad Roshankhah ${ }^{1, *}$, Arman K. Nejad ${ }^{2}$, Orlando Teran ${ }^{3}$, and Kami Mohammadi ${ }^{1}$ \\ ${ }^{1}$ California Institute of Technology, 1200 E. California Blvd., Pasadena, CA, USA \\ ${ }^{2}$ Independent Consultant, Houston, TX, USA \\ ${ }^{3}$ Ovintiv Inc., The Woodlands, TX, USA
}

\begin{abstract}
In this study, we present the results of two-dimensional numerical simulations for the effects of rock matrix permeability on the behaviour of hydraulic fractures in intact and pre-fractured rocks. The simulations are performed using the Finite-Discrete Element Method (FDEM). In this method, the deformation and fluid pressure fields within the porous rock blocks, pre-existing fracture network, and hydraulically induced fractures are calculated through a fully coupled hydromechanical scheme. Furthermore, new fractures can initiate in crack elements located between each pair of finite elements and can propagate in any path that the boundary and loading conditions require according to non-linear fracture mechanics criteria. Fluid channels are also defined between pairs of finite elements simulating the interconnected flow paths through porous media. Four models of the rock mass are created in this study: (i) homogeneous-impermeable, (ii) homogeneous-permeable, (iii) heterogeneous-impermeable matrix, and (iv) heterogeneous-permeable matrix. Heterogeneous rock masses contain a discrete fracture network (natural fractures) in the rock mass structure. Hydraulic fracturing is modelled in domains of $40 \times 40 \mathrm{~m}^{2}$ with the four different structures and mass transport capacities, and the results are compared to each other. The results highlight the significant effect of diffusive fluid flow through rock blocks, in addition to the flow through fracture network, on the global hydromechanical behaviour of the rock mass. These results help to understand the governing hydromechanical processes taking place in fractured rocks with matrix of different permeability, such as granites, shales, carbonate rocks, and sandstones and the extent of complexities required to model their behaviour to achieve reasonable accuracy.
\end{abstract}

\section{Introduction}

Hydraulic fracturing is a technology to enhance the production rate of hydrocarbon fluids and heat from low permeability rock formations [2]. The accuracy of models to design this process depends on how close the models are to the real system properties and boundary conditions. Many theoretical and experimental studies are conducted to understand the behaviour of hydraulic fractures (HFs) in rocks as complex natural materials manifested in their Discontinuous, Inhomogeneous, Anisotropic, Non-Elastic (DIANE) [9], and permeable structure. It is known that hydromechanical behaviour of rocks depends on the interactions between HFs and the natural fracture (NF) network as well as the interactions between the solid skeleton, involved fluids, and boundary conditions [6]. One of the important properties of rocks affecting their hydromechanical behaviour is also their mass transport capacity. Matrix permeability and the equivalent rock permeability strongly correlate with the magnitude of built up pressure upon fluid injection in a poro-elastic medium [18].
Research shows that flow channelling occurs in fractured rocks (especially, in open fractures), i.e., preferred pathways with the least resistance carry most of the fluid [22], while most of the storage occurs in the matrix surrounding those main fractures $[15$ and references therein]. The governing regimes of fluid flow in fractured rocks strongly depend on geometric characteristics of the fracture network (fractures orientation, length, width, aperture, spacing, surface roughness), permeability of the rock matrix, and fluid flow characteristics (density, viscosity, imposed flow rate or pressure gradient). The contribution of each permeable solid component (matrix and fracture network) to flow, however, is not simply proportional to its permeability; rather, a more complicated coupling between the compressibility, porosity, permeability, and shape of each component affect the processes. Solving convection-diffusion equation for a simplified configuration of matrix-fracture system (e.g., in series) reveals that matrix flow weakens the fracture flow contribution, and these effects appear as the matrix-tofracture permeability ratio exceeds only 0.01 [25].

\footnotetext{
* Corresponding author: $\underline{\text { shroshan } @ \text { caltech.edu }}$
} 
Planar circular hydraulic fractures form in both impermeable and permeable homogeneous and isotropic media under anisotropic stress condition, i.e., the HF plane is perpendicular to the minimum principal stress, and it propagates parallel to the maximum and intermediate principal stresses. HFs in a homogeneous medium under isotropic stress have a more complex pattern, i.e., they are shorter and multi-branched [12]. While the HF pattern/shape in homogeneous media is consistent across various studies, there is a discrepancy about the size and growth rate of HF induced in media with different mass transport capacities. On the one hand, some studies show that longer natural HFs form in more permeable rocks because the solid skeleton does not deform as much as that in the low permeability rocks [3]. On the other hand, other studies predict that wider but shorter HFs, which require larger pressure, initiate in high permeability rocks [18]. At any specific time after the start of fluid injection with constant flow rate, longer fractures form in a lower permeability medium while a narrower area around the main fracture in the rock matrix is influenced by the fracturing fluid [11].

HF behaviour in fractured rocks is totally different than that in intact cohesive, homogeneous media, even with the same bulk permeability, because NFs significantly disturb the stress and pressure fields [5, 24]. Orientation and magnitude of the present-day in-situ principal stresses and the type, pattern, and orientation of NFs govern the behaviour of hydraulic fractures in natural rocks [6]. Reactivation of narrow mineralized NFs [5], shear-induced dilation of critically-oriented NFs [27], rock plastic deformations [8, 23], and self-propped NFs due to kinematic dilation of the surrounding rock blocks $[16,17]$ are possible mechanisms for the creation of larger producing volumes (dry stimulated reservoir volume) [14], higher breakdown pressures than the industry-standard models predict [18], and the success of slick water (no proppant) with high flow rate HF treatments in pre-fractured formations [24].

Understanding and predicting the behaviour of such a multi-phase, multi-scale system is still an open question. Particularly, the role of rock matrix permeability in the evolving hydromechanical properties of rocks subjected to $\mathrm{HF}$ is investigated in this study. We utilize a fully coupled hydromechanical FDEM simulation tool called Irazu [7] to study the variation in the behaviour of hydraulic fractures in naturally-fractured rock masses with permeability of the rock matrix.

\section{Numerical modelling}

\subsection{Mathematical framework}

FDEM formulation combines principles of continuum mechanics with discrete element interaction algorithms. With this scheme, FDEM can capture the transition of a rock mass from an initial semi-continuum state to a semi-discrete state when it is subjected to various hydromechanical excitations. In this formulation, the problem domain is first discretized with three-node finite elements, which deform based on the assigned elastic properties (e.g., Young's modulus and Poisson's ratio) but do not break. Between each pair of finite elements, a four-node crack element is embedded, which can break if the resultant stresses on it exceed the assigned strength properties (tensile strength, friction coefficient, cohesion, and fracture energy). Fig. 1a is a schematic view of the discretized domain.

(a)

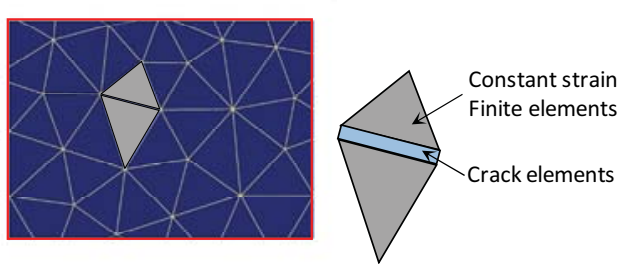

(b)

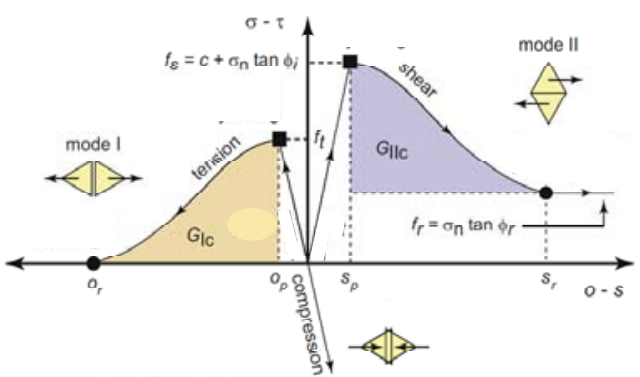

(c)

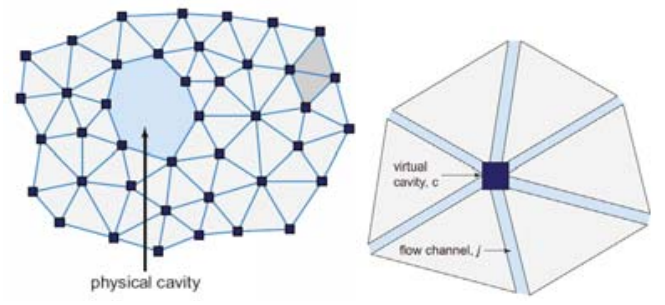

Fig. 1. Schematic representation of FDEM formulation. (a) The domain is discretized by three-node finite elements interfaced by four-node crack elements. (b) Nonlinear fracture mechanics criteria for the response of crack elements in opening and/or shear failure modes (c) Flow channels along the interface elements connect the virtual cavities at the nodes of finite elements and physical cavities if exist (after [11]).

An explicit second order finite difference time integration scheme is used to solve the equation of motion $u(\mathrm{~m})$ for the defined elements with lumped mass $M(\mathrm{~kg})$ and damping $C(\mathrm{~kg} / \mathrm{s})$ diagonal tensors [7].

$$
M \ddot{u}+C \dot{u}+F_{\text {int }}(u)=\bar{F}_{\text {ext }}(u)
$$

Resultant internal forces $F_{\text {int }}(\mathrm{N})$ and external forces $\bar{F}_{\text {ext }}(\mathrm{N})$ in this formulation involve two components more than the ones involved in conventional finite element formulations, which normally include the forces due to internal elastic deformations $F_{e}(\mathrm{~N})$, forces due to external mechanical loads $F_{\text {ext }}(\mathrm{N})$, and forces due to imposed fluid pressure $F_{f l}(\mathrm{~N})$. The additional components include the crack element bonding forces $F_{b}$ $(\mathrm{N})$ and the contact forces $F_{c}(\mathrm{~N})$ between the separated bodies:

$$
\begin{gathered}
F_{\text {int }}(u)=F_{e}(u)+F_{b}(u) \\
\bar{F}_{\text {ext }}(u)=F_{\text {ext }}(u)+F_{f l}(u)+F_{c}(u)
\end{gathered}
$$


The bonding force is calculated according to the nonlinear fracture mechanics model for each fracture type, either opening or shearing or a combination of both, and as a function of crack displacement in the intrinsic cohesive fracture process zone as shown by $o$ (opening distance) and $s$ (shearing distance) in Fig. 1b. If the stresses in a crack element reach its shear or tensile strength, it fails [13]. The evolution of crack opening depends on crack fracture energy in each fracturing mode.

Once a crack element is broken, the separated bodies are treated as discrete elements, and contact forces are calculated for them. The contact force has two components: the repulsive force $F_{r e p}(\mathrm{~N})$ and the frictional force $F_{f r i}(\mathrm{~N})$.

$$
F_{c}(u)=F_{\text {rep }}(u)+F_{f r i}(u)
$$

The repulsive force is calculated using the penalty function method by integrating an infinitesimal repulsive force (due to an infinitesimal overlapping area), which is the difference of the gradients of the potential functions $\varphi$ of the target ( $\mathrm{t}$ ) and contacting (c) elements [13].

$$
F_{\text {rep }}(u)=\sum_{i=1}^{n} \sum_{j=1}^{m} \int_{\beta_{t} \cap \beta_{c}}\left[\nabla \phi_{c j}\left(P_{c}\right)-\nabla \phi_{t i}\left(P_{t}\right)\right] d A
$$

The contact stiffness is introduced through the normal and tangential penalty functions and the overlapping area is the common surface perimeter of the two contacting bodies $\beta_{t} \cap \beta_{c}$. The frictional force is obtained from Coulomb's friction law, i.e., the two discrete elements in contact would experience a relative shear slip if the shear forces applied on the contact walls exceed the product of friction coefficient and normal force applied on the walls.

$$
F_{f r i}(u)=\min \left[p_{t}|s|,\left|\sigma_{n} \tan \varphi_{f}\right|\right] d A
$$

In the hydraulic solver, the elements between finite elements play the role of fluid channels that connect virtual cavities at the nodes of triangular finite elements to each other and create an interconnected network as shown in Fig. 1c. These channels also connect to physical cavities like boreholes. According to cubic law for the flow of a Newtonian fluid with viscosity $\eta$ (Pa.s) in a parallel plate channel with aperture $e(\mathrm{~m})$, width $W$ (m) and length $L(\mathrm{~m})$, the flow rate $q\left(\mathrm{~m}^{3} / \mathrm{s}\right)$ is $[19,26]$

$$
q=-\frac{W e^{3}}{12 \eta} \frac{\Delta P}{L}
$$

An explicit time integration scheme is used in the hydraulic solver as well, which makes the hydromechanical coupling and parallel programming formulations simpler. At each hydraulic time step, the fluid pressure is computed in all channels, which is then used as an inter-elemental surface force boundary condition in the mechanical solver. Natural fractures are explicitly defined in the domain. In general, the finite elements are randomly oriented, however, the edges of finite elements are aligned along the discrete fractures.

\subsection{Model configurations and properties}

Fluid injection at the center of a $40 \times 40 \mathrm{~m}^{2}$ domain is modelled. The borehole geometry is not included, instead, water with a constant flow rate $\mathrm{q}=0.41 / \mathrm{s} / \mathrm{m}$ is injected in a virtual cavity at the centre (Neumann boundary condition). The external boundaries of the model are fixed (zero displacement), and absorbing boundaries are applied to dissipate the dynamic effects of loading. The in-situ stress state is $\sigma_{v}=102 \mathrm{MPa}$ and $\sigma_{h}=58 \mathrm{MPa}[1]$, and the geostatic stress equilibration is achieved with an initial FEM simulation in 200,000 time-steps $(\Delta \mathrm{t}=0.97 \mu \mathrm{s})$. Four models are constructed: (1) homogeneous-impermeable (HoIm), (2) homogeneous-permeable (HoPe), (3) heterogeneousimpermeable matrix (HeIm), (4) heterogeneouspermeable matrix (HePe). Figure 2 shows a schematic view of the models in this study.

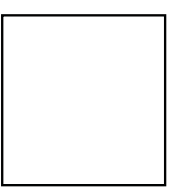

(a) HoIm

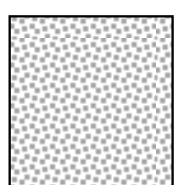

(b) $\mathrm{HoPe}$

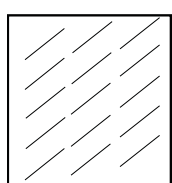

(c) HeIm

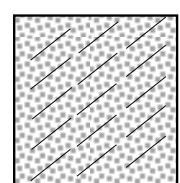

(d) $\mathrm{HePe}$
Fig. 2. Schematic view of the four models constructed in this study. (a) homogeneous-impermeable, (b) homogeneouspermeable, (c) heterogeneous-impermeable matrix, (d) heterogeneous-permeable matrix.

All models are discretized with about 22,000 triangular finite elements. The element size is $0.2 \mathrm{~m}$ in an area of $10 \times 10 \mathrm{~m}^{2}$ centred on the injection point, and it gradually increases to $0.5 \mathrm{~m}$ towards external boundaries. Fig. 3 shows the typical mesh and discrete fracture network (DFN) configuration of homogeneous and heterogeneous models in this study. The models are run for 50,000 steps of mechanical FDEM to establish all overlapping contacts between triangular finite elements under the initial stress field. $35-45 \mathrm{~s}$ of injection is modelled. Fluid leak-off is not considered in these simulations.

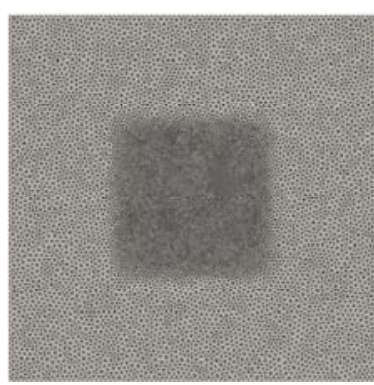

(a) Homogeneous

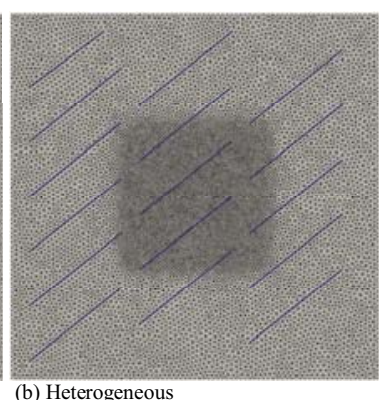

(b) Heterogeneous
Fig. 3. Typical mesh and DFN configurations for: (a) homogeneous media, and (b) heterogeneous media.

Material properties are listed in Table 1. These parameters are selected based on the results of laboratory experiments and analytical or empirical estimations on Eagle Ford shale [20, 21]. The crack energies in mode I \& II and normal \& tangential penalties are calibrated during the numerical calibration procedure to match the 
material behaviour with that observed in a laboratory unconfined compressive strength test on this shale rock $(\mathrm{E}=31 \mathrm{GPa} \& \mathrm{UCS}=25 \mathrm{MPa})$. The initial aperture of hydraulic channels and natural fractures are selected such that the bulk permeability of the model matches with the bulk permeability of this rock $\left(\mathrm{K}=2 \times 10^{-15} \mathrm{~m}^{2}\right)$. This is achieved by conducting several digital permeability tests [11].

Table 1. Material properties used in the numerical simulations.

\begin{tabular}{|c|c|c|c|}
\hline & $\begin{array}{l}\text { Model } \\
\text { components }\end{array}$ & Properties & Values \\
\hline \multirow{13}{*}{$\begin{array}{l}\frac{n}{0} \\
\overline{0} \\
\equiv \\
\equiv\end{array}$} & $\begin{array}{l}\text { Finite } \\
\text { elements }\end{array}$ & $\begin{array}{l}\text { Young's Modulus (GPa) } \\
\text { Poisson's Ratio } \\
\text { Bulk Mass Density }\left(\mathrm{kg} / \mathrm{m}^{3}\right)\end{array}$ & $\begin{array}{c}30 \\
0.25 \\
2500\end{array}$ \\
\hline & \multirow{7}{*}{$\begin{array}{l}\text { Crack } \\
\text { elements }\end{array}$} & Tensile Strength (MPa) & 6 \\
\hline & & Friction Angle $\left({ }^{\circ}\right)$ & 31 \\
\hline & & Cohesion (MPa) & 3.6 \\
\hline & & Mode-I Crack Energy (N.m) & 240 \\
\hline & & Mode-II Crack Energy (N.m) & 2400 \\
\hline & & $\begin{array}{l}\text { Normal Fracture Penalty } \\
(\text { GPa.m) }\end{array}$ & 300 \\
\hline & & $\begin{array}{l}\text { Tangential Fracture Penalty } \\
(\mathrm{GPa} / \mathrm{m})\end{array}$ & 300 \\
\hline & \multirow{3}{*}{ Fluid } & Kinematic Viscosity (Pa.s) & 0.001 \\
\hline & & Mass Density $\left(\mathrm{kg} / \mathrm{m}^{3}\right)$ & 1000 \\
\hline & & Bulk Modulus (GPa) & 0.4 \\
\hline & $\begin{array}{l}\text { Hydraulic } \\
\text { boundary }\end{array}$ & Constant flow rate $\left(\mathrm{m}^{3} / \mathrm{s} / \mathrm{m}\right)$ & $4 \times 10^{-4}$ \\
\hline & $\begin{array}{l}\text { Mechanical } \\
\text { boundary }\end{array}$ & $\begin{array}{l}\text { Pin and absorbing } \\
\text { Vertical \& horizontal in-situ } \\
\text { stresses (MPa) }\end{array}$ & $\begin{array}{c}\varepsilon_{\mathrm{x}}=\varepsilon_{\mathrm{y}}=0 \\
\sigma_{\mathrm{v}}=102, \\
\sigma_{\mathrm{h}}=58\end{array}$ \\
\hline \multirow{6}{*}{ 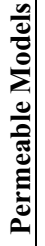 } & & Matrix Permeability $\left(\mathrm{m}^{2}\right)$ & $2 \times 10^{-15}$ \\
\hline & & Initial Aperture with DFs (m) & $1.2 \times 10^{-5}$ \\
\hline & Hydraulic & Initial Aperture without DFs (m) & $1.6 \times 10^{-5}$ \\
\hline & channels & Residual Aperture (m) & $10^{-4}$ \\
\hline & & Aperture Threshold (m) & $4 \times 10^{-4}$ \\
\hline & & Liquid Saturation & 1 \\
\hline \multirow{8}{*}{ 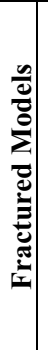 } & \multirow{8}{*}{$\begin{array}{l}\text { Discrete } \\
\text { Fractures }\end{array}$} & Tensile Strength (MPa) & 1 \\
\hline & & Friction Angle $\left({ }^{\circ}\right)$ & 31 \\
\hline & & Cohesion $(\mathrm{MPa})$ & 0.6 \\
\hline & & Mode-I Crack Energy (N.m) & 24 \\
\hline & & Mode-II Crack Energy (N.m) & 240 \\
\hline & & $\begin{array}{l}\text { Normal Fracture Penalty } \\
\text { (GPa.m) }\end{array}$ & 300 \\
\hline & & $\begin{array}{l}\text { Tangential Fracture Penalty } \\
(\mathrm{GPa} / \mathrm{m})\end{array}$ & 300 \\
\hline & & Initial Aperture (m) & $4 \times 10^{-5}$ \\
\hline
\end{tabular}

\section{Results and discussion}

Fig. 4 presents the fluid pressure distribution in the four models, and Fig. 5 shows the mode of induced fractures. In the homogeneous-impermeable medium (HoIm), two hydraulic fractures form propagating upward and downward from the injection point and parallel to the maximum principal stress (vertical direction). The highest pressure is concentrated in the main fracture, and only a very narrow area around HFs is influenced by the injected fluid. The pattern, length, mode, and maximum pressure of $\mathrm{HFs}$ in $\mathrm{HoPe}$ are different than those in HoIm (Fig. 4 and 5a \&b). That is, the mobilized pressure is higher in the permeable medium, but HFs are shorter, they are oriented in vertical/sub-vertical directions, and propagate in mode I and II. In HoPe, the water can flow through all hydraulic channels connected to the injection point, thus, the crack elements fail in vertical/sub-vertical directions due to the mobilized fluid pressure in combination with the farfield mechanical stress. In the impermeable medium (HoIm), the two HFs advance in the toughnessdominated regime because the viscous fluid leak-off and storage regimes do not exist. In the permeable medium (HoPe), however, part of the input energy is expended in the flow through porous medium and influencing a larger area by diffusion; therefore, shorter HFs form in HoPe.
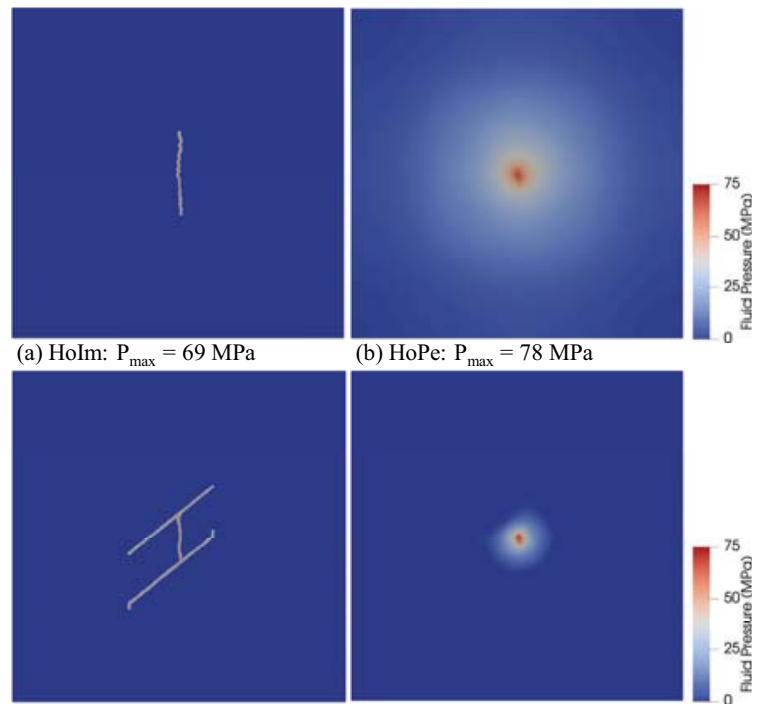

(b) HoPe: $\mathrm{P}_{\max }=78 \mathrm{MPa}$

(c) HeIm: $\mathrm{P}_{\max }=70 \mathrm{MPa}$

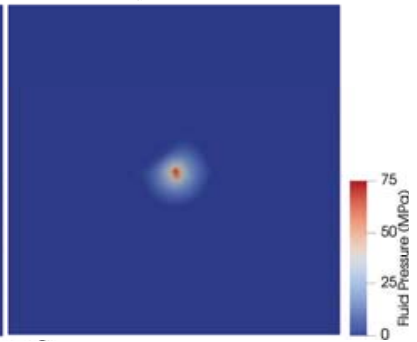

(d) HePe: $\mathrm{P}_{\max }=73 \mathrm{MPa}$

Fig. 4. Pressure distribution for four models. (a) homogeneous-impermeable, (b) homogeneous-permeable, (c) heterogeneous-impermeable matrix, (d) heterogeneouspermeable matrix.

In the heterogeneous media (HeIm and HePe), like homogeneous media, HFs tend to initiate first as bi-wing planar cracks and propagate in mode-I (Fig. 4 and 5c \& d); note that the injection point is in the intact matrix part. In HeIm, HFs propagate in mode-I until they reach the first natural fractures at the top and bottom (Figure $5 \mathrm{c})$. The approach angle of HF/NF, state of in-situ stress, and injection parameters (fluid viscosity and flow rate) in HeIm result in the diversion of HFs into the intersected NFs instead of crossing them. HFs activate NFs (slip), then, HFs continue propagating parallel to major principal stress from the tips of the bottom NF.

In HePe, however, a couple of very short HFs form in the bulk matrix; the fluid then diffuses through the porous matrix and reaches the first NF at the top. By the end of stimulation, several NFs are activated in HePe due to the mechanical stress redistribution. In other words, although HFs are shorter in HePe than in HeIm, HePe's production may not be necessarily less than HeIm because many NFs are still activated and will collect the desorbed gas from the surrounding matrix. 
Fig. 4 and $5 \mathrm{a} \& \mathrm{c}$ show that the impermeable matrix with heterogeneous fabric (HeIm) does not mobilize higher fluid pressure than the homogeneous medium (HoIm). However, the total length of induced discontinuities in HeIm is much higher than HoIm. The discontinuities generated in HeIm include a fracture directly generated by fluid injection in the rock matrix, two NFs activated by the fluid reaching them, and a few parts of other NFs activated only by the mechanical stress redistribution (dry stimulated reservoir).

Fig. 4 and $5 \mathrm{~b} \& \mathrm{~d}$ show that the permeable matrix with heterogeneous fabric $(\mathrm{HePe})$ mobilizes lower fluid pressure than the homogeneous medium (HoPe). The total length of induced discontinuities in $\mathrm{HePe}$ is much higher than HoPe, but almost all the fractures in HePe form away from the injection point (dry stimulated reservoir). This shows the advantage of a fractured reservoir to a homogeneous reservoir, i.e., lower required pressure, but longer induced fractures, which means larger producing area.

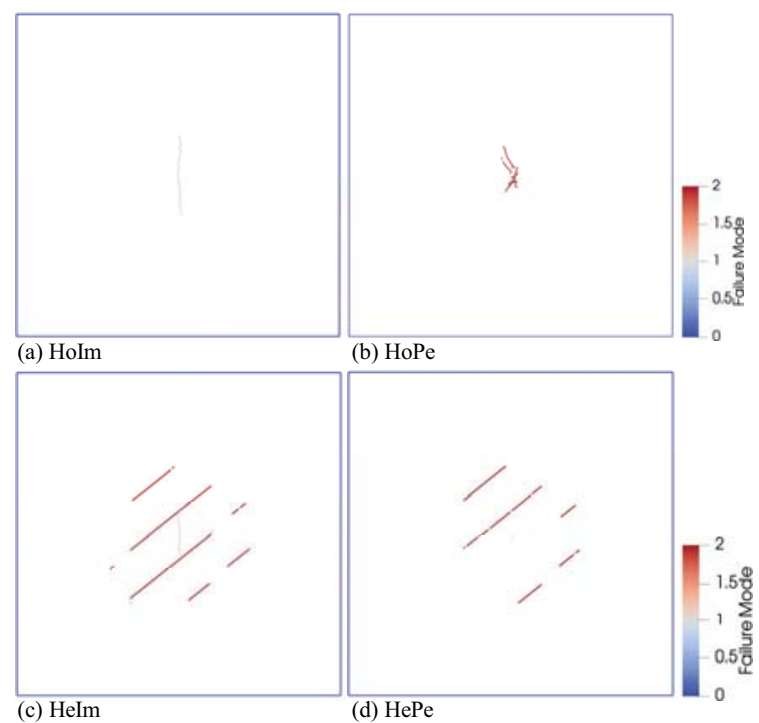

Fig. 5. Mode of fracturing for four models: 1 corresponds to Mode-I or opening, 2 corresponds to mode-II or shearing, and values ranging from 1 to 2 show a mixed-mode fracturing. (a) homogeneous-impermeable, (b) homogeneous-permeable, (c) heterogeneous-impermeable matrix, (d) heterogeneouspermeable matrix

The displacement field of the four models, Fig. 6, shows that the displacement is concentrated around fractures containing the fracturing fluid (Fig. 6a, b \& c). The displacement is, however, more uniformly distributed in the heterogeneous-permeable medium (one-third of other models). The magnitude of displacements is not totally informative about the internal deformation of various media. To understand the stored strain energy in each medium and its implications on the HF propagation, we should inspect the relative displacement of the nodes normalized by the original distance between them (strain field).

The range of strain magnitude is the same for all models as shown in Fig. 7. The magnitude of strain throughout the homogeneous-permeable medium (HoPe) is slightly higher than other models, but this is not the case in the heterogeneous-permeable medium ( $\mathrm{HePe})$.
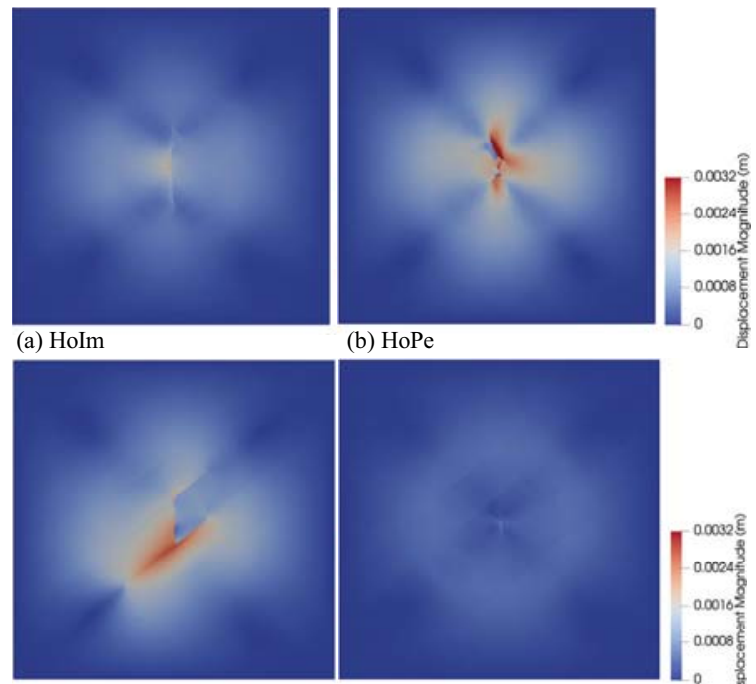

(b) $\mathrm{HoPe}$

(c) HeIm

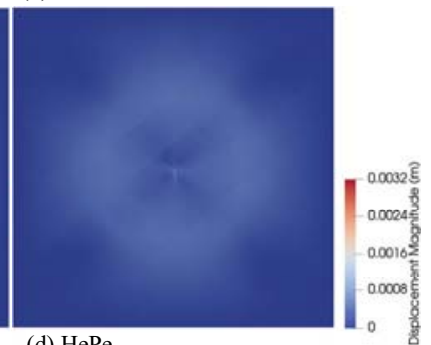

Fig. 6. Displacement field for four models. (a) homogeneousimpermeable, (b) homogeneous-permeable, (c) heterogeneousimpermeable matrix, (d) heterogeneous-permeable matrix.

Therefore, the previous hypothesis that the permeable matrix deforms less than the impermeable medium, and this causes the generation of longer HFs [3], is not validated here. That hypothesis assumes that lower pressure must be mobilized in the permeable medium because the fluid can leak off through the porous matrix, but we obtain higher pressure built-up in the permeable medium. We do not observe longer HFs in permeable media. In the presence of NFs, hardly any HF is generated in the permeable medium, but several NFs activate. These results agree with some of the previous studies $[11,19]$.
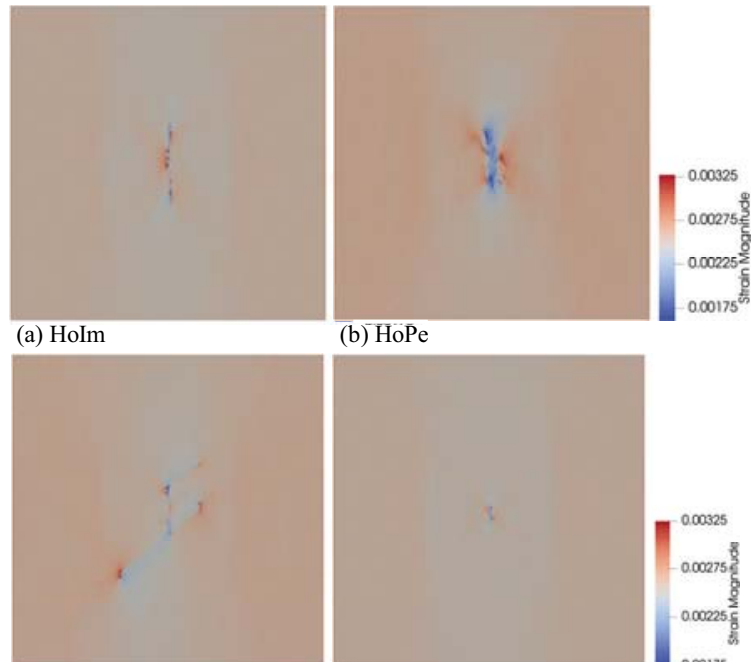

(b) $\mathrm{HoPe}$

(c) HeIm

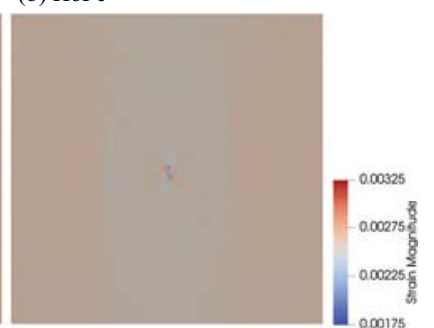

(d) $\mathrm{HePe}$

Fig. 7. Strain magnitude for four models. (a) homogeneousimpermeable, (b) homogeneous-permeable, (c) heterogeneousimpermeable matrix, (d) heterogeneous-permeable matrix. 
Characteristics of the induced HFs and activated NFs are listed in Table 2, including the total length of broken joints $l_{T}$ that consists of the length of cracks failing in mode-II $l_{I I}$ and the length of cracks failing in mode-I/II $l_{I / I I}$, shown in parentheses, average slip distance of modeII fractures $s_{I I}$ with its range in parentheses, and the average opening aperture and slip distance of mode-I/II fractures $e_{I / I I}$ and $s_{I / I I}$ with their ranges in parentheses. Note that no cracks fail in pure mode-I. Heterogeneities (natural fractures) and permeability of the matrix have the same effect in the sense that length of crack elements failed in mode-II is much higher than those failed in mode-I/II.

Table 2. Characteristics of the induced HFs in the four models.

\begin{tabular}{|l|l|l|l|l|}
\hline & HoIm & HoPe & HeIm & HePe \\
\hline$l_{T}\left(l_{I I} \& l_{I / I}\right)$ & $10.31 \quad(0.00$ & 16.34 & 43.73 & 22.19 \\
$(\mathrm{~m})$ & $\& 10.31)$ & $(7.73 \quad \&$ & $(36.04 \quad \&$ & $(21.67 \quad \&$ \\
& & $8.61)$ & $7.69)$ & $0.52)$ \\
\hline Mode-II: $s_{I I}$ & - & $2.2(1.1-$ & $1.6(0.1-$ & $0.1(0.1-$ \\
$(\mathrm{mm})$ & & $4.2)$ & $5.7)$ & $0.1)$ \\
\hline Mode-I/II: & $1.5(0.3-2.9)$ & $0.4(0.0-$ & $1.7(0.0-$ & $0.5(0.4-$ \\
$e_{I / I I} \& \quad s_{I / I I}$ & $\&$ & $4.1) \&$ & $2.7) \&$ & $0.7) \&$ \\
$(\mathrm{~mm})$ & $1.0(0.3-2.2)$ & $1.9(0.1-$ & $0.79(0.0-$ & $0.5(0.1-$ \\
& & $4.5)$ & $2.3)$ & $0.9)$ \\
\hline
\end{tabular}

\section{Conclusions and outlook}

Results of finite-discrete element simulations in this study show that simplifying the hydro-mechanical modelling of rocks as homogeneous and impermeable materials can produce inaccurate predictions. Four cases studied in this numerical analysis show that rock masses respond very differently depending on their structural heterogeneity and matrix permeability. The results agree with the results published in the literature that proves FDEM formulation is capable of modelling HF and underlying processes in various geo-energy systems. Other salient conclusions include:

- Pervious matrix requires higher fluid pressure to fracture because randomly oriented hydraulic channels act as small flaws that concentrate stresses (plastic deformations).

- The stimulated reservoir area is beyond the region that receives the injection fluid either through extended HFs or diffusion through the permeable matrix. When HFs form, they disturb the stress field, then NFs redistribute the stresses and activate (open/slip). Activated NFs form the dry stimulated reservoir area and contribute to the production.

- With the material parameters and DFN configuration used in this study, only a few short HFs form in the heterogeneous-permeable medium (HePe), but several NFs activate as the result of stress redistribution and interactions with the poromechanical properties of the rock.

- Further systematic sensitivity analyses should be performed to understand the regimes imposed by the poromechanical properties of the rock matrix and geometric/statistical characteristics of DFN on the efficiency of hydraulic stimulation.
Authors would like to thank MicroSeismic Inc. for providing the opportunity for this collaboration and Geomechanica Inc. for providing an excellent technical support.

\section{References}

1. A.A. Alalli, Zoback, M. "Microseismic Evidence for Horizontal Hydraulic Fractures in the Marcellus Shale, Southeastern West Virginia," The Leading Edge, Geomech., May (2018)

2. F. Aminzadeh, "Hydraulic Fracturing, An Overview," J. Sus. Energy Eng. 6, 3, 204-228 (2018).

3. D.F. Boutt, Goodwin, L., McPherson, B.J.O.L. "Role of Permeability and Storage in the Initiation and Propagation of Natural Hydraulic Fracture,s" Water Resource Res, 45, W00C13 (2009)

4. M.K. Fisher, Heinze, J.R., Harris, C.D., Davidson, B.M., Wright, C.A., Dunn, K.P. "Optimizing Horizontal Completion Techniques in the Barnett Shale Using Microseismic Fracture Mapping," Proc. Soc. Petro. Eng. Ann. Tech. Conf., Houston, TX, SPE90051 (2004)

5. J.F.W. Gale, Laubach, S.E., Olson, J.E., Eichhubl, P., Fall, A. "Natural fractures in shale: a review and new observations," AAPG Bull., 98, 11, 2165-2216 (2014)

6. J.F.W. Gale, Reed, R.M., Holder, J. "Natural Fractures in the Barnett Shale and their Importance for Hydraulic Fracture Treatments," AAPG Bull., 91, 603, 22 (2007)

7. Geomechanica Inc., 2019. Irazu Software. Theory Manual. Toronto, Canada.

8. L.N. Germanovich, Astakhov, D.K., Shlyapobersky, J., Mayerhofer, M.J., Dupont, C., Ring, L.M. "Modeling Multi-segmented Hydraulic Fracture in Two Extreme Cases: No Leak-off and Dominating Leak-off," Int. J. Rock Mech. Min. Sci., 35, 4-5, 551-554 (1998)

9. J.P. Harrison, Hudson, J.A. Engineering rock mechanics. Part 2: Illustrative workable examples. (P. Sarkka, Eloranta, P. eds. Oxford, 2000)

10. C. Kirsch, "Die Theorie der Elastizitat und die Bedurfnisse der Festigkeitslehre," Zeitschrift Vereines Deutscher Ing., 16, 29, 797-807 (1898)

11. A. Lisjak, Kaifosh, P., He, L., Tatone, B.S.A., Mahabadi, O.K., Grasselli, G. "A 2D, Fully-coupled, Hydromechanical, FDEM Formulation for Modeling Fracturing Processes in Discontinuous, Porous Masses," Comp. Geotech., 81, 1-18 (2017)

12. A. Lisjak, Mahabadi, O.K., Kaifosh, P., Vietor, T., Grasselli, G. "A Preliminary Evaluation of an Enhanced FDEM Code as a Tool to Simulate Hydraulic Fracturing in Jointed Rock Masses.," Rock Engineering and Rock Mechanics: Structures in and on Rock Masses, (Alejano, Perucho, Olalla, and Jimenez eds., Taylor and Francis Group, London, 2014)

13. A. Munjiza, Andrews, K.R.F. "Penalty Function Method for Combined Finite-discrete Element Systems Comprising Large Number of Separate Bodies," Int. J. Numer. Meth. Engng., 49, 1377-1396 (2000)

14. N.B. Nagel, Sanchez-Nagel, M.A., Zhang, F., Garcia, X., Lee, B. "Coupled Numerical Evaluations of the Geomechanical Interactions between a Hydraulic Fracture Stimulation and a Natural Fracture System in Shale Formations," Rock Mech. Rock Eng. 46, 581-609 (2013)

15. National Academy of Engineering (NAE), Characterization, Modeling, Monitoring, and 
Remediation of Fractured Rock (The National Academies Press, Washington, DC, 2015)

16. S. Roshankhah, Cruz, L.G., Shin, H., Lizcano, A., Santamarina, J.C. "Kinematic Dilation during the Hydraulic Stimulation of Pre-fractured Rocks," Geotech. Lett. 9, 3, 186-192 (2019)

17. S. Roshankhah, Mohammadi, K. "Fabric-dependent Hydro-mechanical Behavior of Pre-fractured Rocks," Geocongress, Feb. 25-28, Minneapolis, MN, USA (2020)

18. E. Sarris, Papanastasiou, P. "Modeling of Hydraulic Fracturing in a Poroelastic Cohesive Formation," Int. J. Geomech., 12, 2, 160-167 (2012)

19. D.T. Snow, A parallel plate model of fractured permeable media (PhD thesis, UC Berkeley, 1965)

20. H. Sone, Zoback, M.D. "Mechanical Properties of Shalegas Reservoir Rocks - Part 1: Static and Dynamic Elastic Properties and Anisotropy," Geophysics, 78, 5, D381-392 (2013a)

21. H. Sone, Zoback, M.D. "Mechanical Properties of Shalegas Reservoir Rocks - Part 2: Ductile Creep, Brittle Strength, and their Relation to the Elastic Modulus," Geophysics, 78, 5, D393-402 (2013b)

22. C.F. Tsang, Neretnieks, I. "Flow Channeling in Heterogeneous Fractured Rocks," Reviews of Geophysics, 36, 2, 275-298 (1998)

23. D. B. van Dam, Papanastasiou, P., de Pater, C.J. "Impact of Rock Plasticity on Hydraulic Fracture Propagation and Closure," SPE Prod. Facil., 17, 3, 149-159 (2002)

24. N.R. Warpinski, Kramm, R.C., Heinze, J.R., Waltman, C.K. "Comparison of Single and Dual Array Microseismic Mapping Techniques in the Barnett Shale," Proc. Soc. Petro. Eng. Ann. Tech. Conf., Dallas, TX, SPE95568 (2005)

25. C. Zhongxiang, Jun, Y. "The Behavior of Naturally Fractured Reservoirs Including Fluid Flow in Matrix Blocks," Transport in Porous Media, 2, 147-163 (1987)

26. R.W. Zimmerman, Bodvarsson, G.S. "Hydraulic Conductivity of Rock Fractures," Transport in Porous Media, 23, 1-30 (1996)

27. M.D. Zoback, Reservoir Geomechanics (Cambridge University Press, 2010) 